\title{
First case of fungal rhinosinusitis due to Aspergillus nomius in a child with aplastic anaemia
}

\author{
Yasemin Oz, ${ }^{1}$ Muge Aslan, ${ }^{1}$ Nuray Gundogdu, ${ }^{1}$ Ozcan Bor ${ }^{2}$ \\ ${ }^{1}$ Division of Mycology, Department of Microbiology, and ${ }^{2}$ Division of Haematology and Oncology, Department of \\ Pediatrics, Faculty of Medicine, Eskişehir Osmangazi University, Eskisehir, Turkey
}

\begin{abstract}
Summary
Recently, infections caused by Aspergillus species have increased dramatically. Invasive aspergillosis (IA) is one of the most important causes of morbidity and mortality in immunocompromised patients, such as those with haematological malignancies who undergo chemotherapy, bone marrow and solid organ transplant recipients, and patients with other immunodeficiency. The most common species causing invasive infections include Aspergillus fumigatus, followed by Aspergillus flavus. Aspergillus nomius is an anamorphic species belonging to Aspergillus section Flavi, which currently include 22 species that can be grouped into seven clades (Aspergillus flavus, Aspergillus tamarii, Aspergillus nomius, Petromyces alliaceus, Aspergillus togoensis, Aspergillus leporis and Aspergillus avenaceus) based on morphological characters, sequence data, and extrolite profiles. These species may also produce toxic and carcinogenic aflatoxins. However, Aspergillus nomius is an emerging pathogen as a cause of IA; we found only two reported cases of invasive infection caused by this fungus in literature up till now. We reported a case of fungal rhi-
\end{abstract}

Correspondence: Muge Aslan, Division of Mycology, Department of Microbiology, Faculty of Medicine, Eskişehir Osmangazi University, Eskisehir, Turkey.

Tel.: +902222392979(4550).

E-mail: drmucu@hotmail.com

Key words: Aspergillus nomius, case, rhinosinusitis.

Contributions: the authors contributed equally.

Conflict of interest: the authors declare no potential conflict of interest.

Funding: none.

Received for publication: 2 March 2019.

Revision received: 5 April 2019.

Accepted for publication: 3 July 2019.

${ }^{\circ}$ Copyright: the Author(s), 2019

Licensee PAGEPress, Italy

Microbiologia Medica 2019; 34:8148

doi: $10.4081 / \mathrm{mm} .2019 .8148$

This article is distributed under the terms of the Creative Commons Attribution Noncommercial License (by-nc 4.0) which permits any noncommercial use, distribution, and reproduction in any medium, provided the original author(s) and source are credited. nosinusitis caused by Aspergillus nomius in a child with aplastic anaemia and to our knowledge, it is the first case as an agent of rhinosinusitis. The isolate was identified by sequencing based methods.

\section{Introduction}

In recent years, the importance of Aspergillus species has increased dramatically. Invasive aspergillosis (IA) is one of the most important causes of morbidity and mortality in immunocompromised patients, such as those with haematological malignancies who undergo chemotherapy, bone marrow and solid organ transplant recipients, and patients with other immunodeficiency (1-4). The most common species causing invasive infections include Aspergillus fumigatus, followed by Aspergillus flavus. Aspergillus nomius (A.nomius) is a heterothallic and also sexually reproducing species belonging to Aspergillus section Flavi, which currently include 27 species that can be grouped into seven clades (A. flavus, A tamarii, A. nomius, Petromyces alliaceus, A. togoensis, A. leporis, and $A$. avenaceus) based on morphological characters, sequence data, and extrolite profiles (5). Several of these species are known to produce highly toxic and carcinogenic aflatoxins (6). A. nomius is an emerging pathogen as a cause of IA; we found only two reported cases of invasive infection due to $A$. nomius in literature up till now. We reported a case of fungal rhinosinusitis caused by $A$. nomius in a child with aplastic anaemia; to our knowledge, it is the first case as an etiological agent of rhinosinusitis.

\section{Case Report}

An 11-year-old girl was hospitalized for 22 days with aplastic anaemia and febrile neutropenia. She was receiving corticosteroid and vancomycin, amikacin, imipenem empirically for persistent fever. Microbiological examination of blood, urine, stool and throat swab specimens was performed for three times during her hospitalization. A consolidation area of lung parenchyma was detected on routine chest X-ray and high-resolution computed tomography (HRCT) revealed a single nodule with ground glass sign and atelectasis suggestive of a fungal infection. Intravenous voriconazole was started in addition to empirical antibacterial treatment.

On day 28, pain, hyperaemia and oedema appeared in the left wing of her nose, progressing to a necrotic lesion. On day 29, nasal and sinus biopsies were obtained and sent to Clinical Microbiology 
Laboratory for microbiological evaluations. Patient persisted as febrile on day 34 , when paranasal computed tomography examination consistent with a fungal infection was performed. Considering being mucormycosis, voriconazole treatment switched to liposomal amphotericin B. On the $46^{\text {th }}$ day of hospitalization, posaconazole was added to antifungal treatment because neither radiological nor clinical regression was subsequently detected with amphotericin B therapy. After another 20 days, cranial Magnetic Resonance Imaging (MRI) and HRCT examination showed the fungal infection progression with left maxillary and infraorbital oedema, bone destruction and mucosal thickening. Imipenem was stopped and the therapy switched to piperacillin-tazobactam, amikacin and metronidazole on the 70th day. Furthermore, caspofungin was added to posaconazole on the 72th day. Diffuse fungal progression was detected in cranial, orbital and paranasal MRI on 79th day, and surgical debridement was recommended for maxillary sinus but this latter could not be performed due to the patient's general condition. Nasal and maxillary lesions spread to her hard palate and upper mouth mucosa. Stenotrophomonas maltophilia was isolated from blood culture the 92th day. She died for septic emboli in spite of broad spectrum antibacterial and antifungal therapy the 97 th day of the hospitalization.

\section{Mycological studies}

Nasal and maxillary specimens were used for microscopic examination, that revealed hyaline and septate hyphae, and for microbiological investigation.

The samples were plated on sheep blood agar, McConkey agar, chocolate agar and three Sabouraud dextrose agar (SDA) plates. The SDA plates were incubated at three different temperatures (26, 30 and $37^{\circ} \mathrm{C}$ ) for a period of 7 days. After 48 hours of incubation, filamentous colonies began to grow on all plates. Initially pale yellow-green, the colonies then became velvety to floccose consisting in light orange-brown vegetative mycelium. At maturity, colonies had green, yellow-green granule like loose mycelial mesh in central, yellow-orange colour in periphery and the colony reverse was light yellow-orange on SDA. Microscopically, conidial heads were uniseriate or biseriate, radiate; vesicles were spherical to subspherical. The conidiophores were variable in length, hyaline and echinulate; conidia were spherical to subspherical, echinulate, and variable in size (Figures 1 and 2). Based on macroscopic and microscopic features, the fungal isolate was identified as Aspergillus flavus (7).

All bacterial cultures were negative.

Precise identification of this fungus was made by PCR amplification of D1-D2 region of 28S rRNA gene, and sequencing of the resulting amplicons. The amplification of genomic DNA was carried out by semi-nested PCR. We used broad-range primer pairs ITS1 (5' TCC GTA GGT GAA CCT GCG G) in the first reaction followed by D1 (5' GCA TAT CAA TAA GCG GAG GA) in the second reaction; D2R (5' TTG GTC CGT GTT TCA AGA CG) was used as the reverse primer in both reactions. Also, a segment of the $\beta$-tubulin gene was amplified using primers bT-F (5' CAACTCCTGACCGCTTCTCC 3') and bT-R (5' GACATGACAGCAGAGACCAG3') and a segment of the calmodulin gene was amplified using primers cmd-F (5' TCGTAAGTAGTTATCGTCGT 3') cmd-R (5' ATCATCTCATCAACTTCGTC 3'). DNA sequence was determined using a BigDye Terminator ver5.1 cycle sequencing kit (Applied Biosystems, Foster City, CA) and an ABI 3130 DNA sequencer. Sequence analysis was carried out by BLASTN similarity search at the website of the National Center for Biotechnology Information (http://www.ncbi.nlm.nih.gov/BLAST). A sequence of 534 bp was detected, exhibiting 100\% identity with Aspergillus nomius isolates present in the publicly available GenBank sequence database of NCBI. After identification, viable cultures were deposit- ed at the Westerdijk Fungal Biodiversity Institute, Utrecht, The Netherlands (CBS 142749).

The $35^{\text {th }}$ day, antifungal susceptibility testing of the maxillary and nasal fungal isolate was performed to determine the minimum inhibitory concentrations (MICs) by using Clinical and Laboratory Standards Institute document M38-A2 (8). MICs of amphotericin $\mathrm{B}$, voriconazole, posaconazole, and minimum effective concentrations (MECs) of caspofungin, and anidulafungin were as follows: $4 \mu \mathrm{g} / \mathrm{mL}, 0.25 \mu \mathrm{g} / \mathrm{mL}, 0.25 \mu \mathrm{g} / \mathrm{mL}, 0.12 \mu \mathrm{g} / \mathrm{mL}$, and $0.03 \mu \mathrm{g} / \mathrm{mL}$, respectively.

\section{Discussion}

IA remains one of the major clinical problems in haematological patients, and rapid discrimination among the etiologic agents is very important for definitive diagnosis and patient's treatment strategies. The long-term use of antibiotics and steroids, chronic bacterial sinusitis, underlying diseases such as diabetes mellitus, haematological malignancies, bone marrow or solid organ transplantation and subsequent chemotherapy, and aplastic anaemia constitute high risk factors for invasive fungal infections. Invasive fungal rhinosinusitis (IFRS) is a rare infection characterized by an infiltration by fungal pathogen of the nasal cavity mucosa and paranasal sinuses. It may metastasize to the orbit, cavernous sinus and cerebral parenchyma via the vascular invasion in immunosuppressed subjects, presenting a progressive course. The mortality of IFRS in immunocompromised patients ranges from $50 \%$ to $80 \%$ since early physical findings are non-specific and ambiguous (i.e., nasal obstruction, purulent discharge, and epistaxis) (1-4). In fact, most patients do not have the classic findings in the early phase of infection: the girl of our case presented facial pain, oedema and necrotic lesions on the nose in late stage.

IFRS is largely attributed to Aspergillus spp. and Zygomycetes in patients with stem cell transplantation and haematological disease (3-4). The genus Aspergillus is divided into several sections or complexes that provide 340 species, of which approximately 40 have been reported in human aspergillosis (9). Therefore, the identification of the agent to species level may be clinically important, since $A$. flavus-like species can show variable antifungal susceptibility, and treatment management may be challenged by drug resistance (10,11). However, many Aspergillus species have similar morphological features, and their identification is quite difficult according to phenotypic features (12). In this regard, comparative sequence analysis of one or several gene regions is required for species-level identification within Aspergillus complexes.

A. nomius, described by Kurtzman et al. in 1987, could be more common than expected because it is very difficult to morphologically distinguish it from A. flavus, the second important cause of invasive aspergillosis. Classification of an isolate in the Aspergillus genus can be based on morphological features but the identification of closely related and highly variable species is difficult by conventional criteria $(9,13)$. A. nomius isolates can produce variously sized sclerotia including the originally described indeterminate ones considered characteristic of this species, as well as S-type sclerotia (13). As an aflatoxigenic member of Aspergillus section Flavi, A. nomius can produce both B and $\mathrm{G}$ aflatoxins, similar to Aspergillus parasiticus which cause serious problems worldwide in agricultural commodities (14). In addition, this species is widespread in different countries in the world such as India, Iran, Thailand, Brazil and it has been reported as foodborne or environmental origin (14-17). However, A. nomius was recently isolated from keratitis, onychomycosis and pneumonia in 


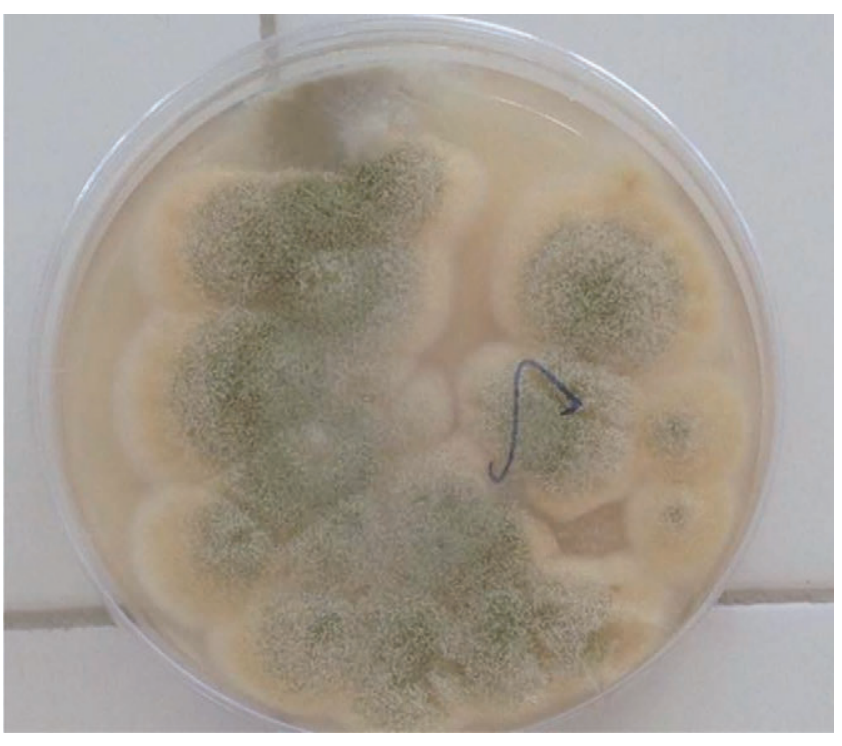

Figure 1. Aspergillus nomius on Sabouraud Dextrose Agar.

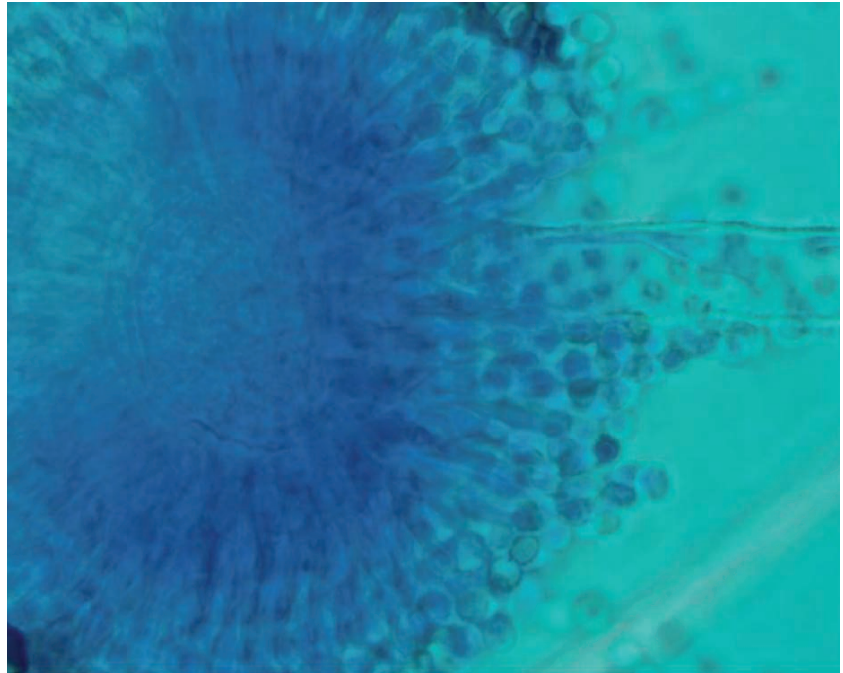

Figure 2. Microscopic appearance of Aspergillus nomius conidial head. humans (18-20). To our knowledge, our patient is the first fungal rhinosinusitis case caused by $A$. nomius in Turkey. Initially this isolate was identified as $A$. flavus based on morphologic characteristics because of the high similarity between these sister species. The final identification was carried out by molecular methods. It is possible that other $A$. nomius isolates have been misidentified as $A$. flavus in our laboratory until now. The differentiation of $A$. nomius from other members of Aspergillus section Flavi can be made by only molecular techniques, including calmoduline, beta tubuline and ITS sequence analysis as it was done in this study $(13,21)$. It should be noted that molecular methods are not applicable in most routine clinical microbiology laboratory.

The present case shows that clinicians should be aware of a possible Aspergillus species when the manifestations of sinusitis appeared in immunocompromised patients, and invasive fungal rhinosinusitis must be taken into consideration. Although A. fumigatus is the most common agent of invasive aspergillosis, rare species such as $A$. nomius may also cause an invasive infection. However, correct identification of uncommon fungal pathogens is difficult for most of clinical microbiology laboratories. Accurate identification of these rare pathogens is important for both epidemiological and management of treatment.

\section{References}

1. Del Gaudio JM, Clemson LA. An early detection protocol for invasive fungal sinusitis in neutropenic patients successfully reduces extent of diseases at presentation and long term morbidity. Laryngoscope 2009;119:180-3.

2. Suslu AE, Ogretmenoglu O, Suslu N, et al. Acute invasive fungal rhinosinusitis: our experience with 19 patients. Eur Arch Otorhinolaryngol 2009;266:77-82.

3. Takahashi H, Hinohira Y, Hato N, et al. Clinical features and outcomes of four patients with invasive fungal sinusitis. Auris Nasus Larynx 2011;38:289-94.

4. Montone KT, Virginia A Livolsi VA, et al. Fungal rhinosinusitis: A retrospective microbiologic and pathologic review of 400 patients at a single university medical center. Int $\mathrm{J}$ Otolaryngol 2012:684835.

5. Horn BW, Moore GG, Carbone I. Sexual reproduction in aflatoxin-producing Aspergillus nomius. Mycologia 2011;103: 174-83.

6. Peterson SW, Ito Y, Horn BW, et al. Aspergillus bombycis, a new aflatoxigenic species and genetic variation in its sibling species, A. nomius. Mycologia 2001;93:689-703.

7. De Hoog GS, Guarro J, Gene J, et al. Atlas of the Clinical Fungi, a pilot CDROM version of the 3rd edition, 2009.

8. Clinical and Laboratory Standards Institute. 2008. Reference Method for Broth Dilution Antifungal Susceptibility Testing of Filamentous Fungi; Approved Standard, 2nd ed.CLSI document M38-A2. Clinical and Laboratory Standards Institute, Wayne, PA.

9. Samson RA, Visagie CM, Houbraken J, et al. Phylogeny, identification and nomenclature of the genus Aspergillus. Stud Mycol 2014;78:141-73.

10. Balajee SA, Lindsley MD, Iqbal N, et al. Nonsporulating clinical isolate identified as Petromyces alliaceus (anamorph Aspergillus alliaceus) by morphological and sequence-based methods. J Clin Microbiol 2007;45:2701-3.

11. Ozhak-Baysan B, Alastruey-Izquierdo A, Saba R, et al. Aspergillus alliaceus and Aspergillus flavus co-infection in an acute myeloid leukemia patient. Med Mycol 2010;48:995-9.

12. Balajee SA, Houbraken J, Verweij PE, et al. Aspergillus species identification in the clinical setting. Stud Mycol 2007;59:39-46.

13. Kurtzman CP, Horn BW, Hesseltine CW. Aspergillus nomius, a new aflatoxin-producing species related to Aspergillus flavus and Aspergillus tamarii. Antonie van Leeuwenhoek 1987;53:147-58.

14. Ehrlich KC, Kobbeman K, Montalbano BG, et al. Aflatoxinproducing Aspergillus species from Thailand. Int $\mathrm{J}$ Food Microbiol 2007;114:153-9.

15. Moody SF, Tyler BM. Restriction enzyme analysis of mitochondrial DNA of the Aspergillus flavus group: A. flavus, A. parasiticus, and A. nomius. Appl Environ Microbiol 1990;56:2441-52. 
16. Razzaghi-Abyaneh M, Shams-Ghahfarokhi M, Allameh A, et al. A survey on distribution of Aspergillus section Flavi in corn field soils in Iran: population patterns based on aflatoxins, cyclopiazonic acid and sclerotia production. Mycopathologia 2006;161:183-92.

17. Olsen M, Johnsson P, Moller T, et al. Aspergillus nomius, an important aflatoxin producer in Brazil nuts? World Mycotoxin J 2008;1:123-6.

18. Manikandan P, Varga J, Kocsube S, et al. Mycotic keratitis due to Aspergillus nomius. J Clin Microbiol 2009;47:3382-85.
19. Zotti M, Machetti M, Persi A, et al. Onychomycosis: first case due to Aspergillus nomius. Acta Derm Venereol 2011;91:5912.

20. Caira M, Posteraro B, Sanguinetti M, et al. First case of breakthrough pneumonia due to Aspergillus nomius in a patient with acute myeloid leukemia. Med Mycol 2012;50:746-50.

21. Hinrikson HP, Hurst SF, Lott TJ, et al. Assessment of ribosomal large-subunit D1-D2, internal transcribed spacer 1, and internal transcribed spacer 2 regions as targets for molecular identification of medically important Aspergillus species. J Clin Microbiol 2005;43:2092-103. 